\title{
GENERALIZED BI-CIRCULAR PROJECTIONS ON MINIMAL IDEALS OF OPERATORS
}

\author{
FERNANDA BOTELHO AND JAMES JAMISON
}

(Communicated by Joseph A. Ball)

\begin{abstract}
We characterize generalized bi-circular projections on $\mathcal{I}(\mathcal{H})$, a minimal norm ideal of operators in $\mathcal{B}(\mathcal{H})$, where $\mathcal{H}$ is a separable infinite dimensional Hilbert space.
\end{abstract}

\section{INTRODUCTION}

The existence of classes of projections on a given Banach space, as well as their characterizations, are basic problems in Banach Space Theory; see [2], [3], [9], [13], and [16]. Recently, a class of projections, namely bi-circular projections, was proposed by Stachó and Zalar in [17]. A projection is called a bi-circular projection if $e^{i \alpha} P+e^{i \beta}(I-P)$ is an isometry for all $\alpha, \beta \in R$. Such projections have been studied in a variety of settings by Stachó and Zalar; see [18]. Fosner, Ilisevic, and C. K. Li, in [7], considered a generalization of this concept by requiring that $P+\lambda(I-P)$ is an isometry, for some $\lambda$ with $|\lambda|=1$. We call these projections generalized bi-circular projections. In this paper, we completely characterize generalized bi-circular projections on minimal norm ideals of Hilbert space operators. It is an easy consequence of our characterization that these projections are bi-contractive. We start by recalling the basic definitions and results to be used throughout the paper.

Definition 1.1. We consider a Banach space $X$ with the norm $\|\cdot\|$. The operator $Q$ (on $X$ ) is said to be a generalized bi-circular projection if and only if $Q^{2}=Q$ and there exists $\lambda \in C, \lambda \neq 1$, and $|\lambda|=1$, for which $Q+\lambda(I-Q)$ is an isometry of $X$, denoted by $\tau$.

We observe that $\tau$ is a surjective isometry. In fact, if $\omega \in X$, there exists $z \in X$, namely $z=Q(\omega)+\frac{1}{\lambda}(\omega-Q(\omega))$, such that $\tau(z)=\omega$.

Let $\mathcal{H}$ be a complex separable Hilbert space of infinite dimension and $\mathcal{B}(\mathcal{H})$ the algebra of bounded linear operators on $\mathcal{H}$. A symmetric norm ideal, $(\mathcal{I}, \nu)$, in $\mathcal{B}(\mathcal{H})$ consists of a two-sided proper ideal $\mathcal{I}$ together with a norm $\nu$ on $\mathcal{I}$ satisfying the conditions:

(i) $\nu(A)=\|A\|$, for every rank 1 operator $A$.

(ii) $\nu(U A V)=\nu(A)$, for every $A \in \mathcal{I}$ and unitary operators $U$ and $V$ on $\mathcal{H}$.

Received by the editors October 16, 2006 and, in revised form, February 15, 2007.

2000 Mathematics Subject Classification. Primary 47A65; Secondary 47B15, 47B37.

Key words and phrases. Isometry, generalized bi-circular projections, Banach spaces, ideals of operators. 
If the set of finite rank operators is dense in $\mathcal{I}$, then $\mathcal{I}$ is a minimal norm ideal; see $[11]$.

The isometries of minimal norm ideals were characterized by Sourour, in [12]. For completeness of exposition we state Sourour's result. First, we recall the concept of transpose of an operator relative to a fixed orthonormal basis, $\left\{e_{i}\right\}$, for the Hilbert space. We denote by $T^{t}$ the transpose of the operator $T$.

Definition 1.2. Given $T \in B(\mathcal{H})$, the transpose $T^{t}$ is defined to be the unique operator in $B(\mathcal{H})$ such that

$$
\left\langle T^{t} e_{i}, e_{j}\right\rangle=\left\langle T e_{j}, e_{i}\right\rangle .
$$

Theorem 1.3 (Sourour [12]). If $\mathcal{I}$ is a minimal ideal in $B(\mathcal{H})$ different from $C_{2}(\mathcal{H})$, and $\mathcal{U}$ is a linear transformation on $\mathcal{I}$, then $\mathcal{U}$ is a surjective isometry of $\mathcal{I}$ if and only if there exist unitaries $U$ and $V$ on $\mathcal{H}$ such that

$$
\mathcal{U}(T)=U T V \quad \text { or } \mathcal{U}=U T^{t} V
$$

for every $T \in \mathcal{I}$.

In addition to the result of Sourour, the following theorem due to Fong and Sourour (cf. [15]) will also be used in our proofs.

The operators $\left\{A_{i}\right\}_{i=1, \ldots, m}$ and $\left\{B_{i}\right\}_{i=1, \ldots, m}$ are bounded operators on the Banach space $X$ and $\Phi$ acts on $B(X)$ as follows:

$$
\Phi(T)=A_{1} T B_{1}+A_{2} T B_{2}+\cdots+A_{m} T B_{m} .
$$

Theorem 1.4 (Fong and Sourour [15]). If $\Phi(T)=O$, for all $T \in B(X)$, then $\left\{B_{1}, B_{2}, \cdots, B_{m}\right\}$ is linearly dependent. Furthermore, if $\left\{B_{1}, B_{2}, \cdots, B_{n}\right\}(n \leq m)$ is linearly independent, and the $\left(c_{k j}\right)$ denote constants for which

$$
B_{j}=\sum_{k=1}^{n} c_{k j} B_{k}, \quad n+1 \leq j \leq m,
$$

then $\Phi(T) \equiv 0$ for all $T \in B(X)$ if and only if

$$
A_{k}=-\sum_{j=n+1}^{m} c_{k j} A_{j}, \quad 1 \leq k \leq n .
$$

If $n=m$, then $A_{1}=A_{2}=\cdots=A_{m}=0$.

\section{Generalized Bi-Circular projections ON IDEALS OF HILBERT SPACE OPERATORS}

In [4], the authors have shown that generalized bi-circular projections on certain Banach spaces are the average of the identity with an isometric reflection, i.e. an isometry $R$ such that $R^{2}=I d$. We show in this paper that a similar characterization holds for ideals of Hilbert space operators. In anticipation of this result, we now give a simple characterization of isometric reflections on minimal norm ideals.

Lemma 2.1. The operator $\tau$ on $\mathcal{I}$ is an isometric reflection if and only if either

(1) $\tau(T)=U T^{t} V$ with $U$ and $V$ unitary operators on $\mathcal{H}$ so that $V= \pm\left(U^{t}\right)^{*}$, or

(2) $\tau(T)=U T V$, with $U$ and $V$ isometries of the form $U=\sqrt{\bar{\alpha}} P_{0}-\sqrt{\bar{\alpha}}\left(I d-P_{0}\right)$ and $V=\sqrt{\alpha} P_{1}-\sqrt{\alpha}\left(I d-P_{1}\right)$, where $P_{0}$ and $P_{1}$ are projections onto closed subspaces of $\mathcal{H}$ and $\alpha$ is a complex number of modulus 1 . 
Proof. This lemma is a straightforward consequence of Fong and Sourour's theorem. If $\tau(T)=U T^{t} V$, then $\tau^{2}=I d$ if and only if $U V^{t} T U^{t} V-T=0$, for all $T \in \mathcal{I}$. Therefore, $U^{t} V=\alpha I d$, for some complex number $\alpha$ of modulus 1 , and $V U^{t}=\bar{\alpha} I d$. This implies that $\alpha=\bar{\alpha}$ and hence $\alpha= \pm 1$. Consequently $V= \pm\left(U^{t}\right)^{*}$.

If $\tau(T)=U T V$, then $\tau^{2}=I d$ if and only if $U^{2} T V^{2}-T=0$, for all $T \in \mathcal{I}$. This implies that $V^{2}=\alpha I d$, with $|\alpha|=1$, and $U^{2}=\bar{\alpha} I d$. The second statement now follows from the spectral theorem applied to $U$ and $V$; see [14].

Proposition 2.2. Let $(\mathcal{I}, \nu)$ be a separable minimal norm ideal different from $\mathcal{C}_{2}$ (the Hilbert-Schmidt class) and let $Q$ be a generalized bi-circular projection on $\mathcal{I}$. If $Q$ is associated with a surjective isometry $\tau$ on $\mathcal{I}$ of the form $\tau(T)=U T^{t} V$ ( $U$ and $V$ unitary operators on $\mathcal{H})$, then $Q$ is the average of the identity with an isometric reflection.

Proof. If $Q$ is a generalized bi-circular projection, then

$$
Q(T)=\frac{1}{1-\lambda}\left[-\lambda T+U T^{t} V\right]
$$

and

$$
\lambda T-(\lambda+1) U T^{t} V+U V^{t} T U^{t} V=O,
$$

for every $T \in \mathcal{I}$. We first observe that for $\lambda=-1$, the equation (2.1) reduces to

$$
-T+U V^{t} T U^{t} V=O, \text { for all } T \in \mathcal{I} \text {. }
$$

Fong and Sourour's theorem implies that $U^{t} V=\alpha I d$ and $I d=\alpha U V^{t}$, for some modulus 1 complex number $\alpha$. Therefore $\alpha^{2}=1$ and $V= \pm U^{* t}$. The projection $Q$ is the average of the identity with the isometric reflection: $R(T)= \pm U T^{t} U^{* t}$. Now, we consider $\lambda \neq-1$. We show that there are no unitary operators $U$ and $V$ for which the equation (2.1) holds for every $T \in \mathcal{I}$.

We fix $\lambda$, with modulus 1 and different from -1 . If there exists a pair of unitary operators $(U, V)$ so that equation (2.1) holds for every $T \in \mathcal{I}$, then

$$
T^{t}=\frac{1}{\lambda+1} U^{*}\left[\lambda T+U V^{t} T U^{t} V\right] V^{*}
$$

and

Therefore

$$
T=\frac{1}{\lambda+1} V^{* t}\left[\lambda T^{t}+V^{t} U T^{t} V U^{t}\right] U^{* t} .
$$

$$
T=\frac{1}{(\lambda+1)^{2}}\left[\lambda^{2} V^{* t} U^{*} T V^{*} U^{* t}+2 \lambda T+U V^{t} T U^{t} V\right]
$$

or equivalently

$$
-\left(\lambda^{2}+1\right) T+\lambda^{2} V^{* t} U^{*} T V^{*} U^{* t}+U V^{t} T U^{t} V=O, \forall T \in \mathcal{I} .
$$

Fong and Sourour's theorem implies that $\left\{I d, V^{*} U^{* t}, U^{t} V\right\}$ is linearly dependent and we consider the following cases:

I. $U^{t} V=\alpha I d$; then $V^{*} U^{* t}=\bar{\alpha} I d$ and $V=\alpha U^{* t}$. Equation (2.1) becomes

$$
\left(\lambda+\alpha^{2}\right) T-(\lambda+1) \alpha U T^{t} U^{* t}=O .
$$

This also implies that $\left(\frac{\lambda+\alpha^{2}}{(\lambda+1) \alpha}\right)^{2}=1$ and $T U^{t}= \pm U T^{t}, \forall T \in \mathcal{I}$. We show that this is impossible. We first consider $T=e_{i} \otimes e_{j}$, with $i \neq j$. Therefore, we have that $T U^{t}\left(e_{k}\right)=\left\langle U^{t}\left(e_{k}\right), e_{j}\right\rangle e_{i}= \pm U T^{t}\left(e_{k}\right)= \pm\left\langle e_{k}, e_{i}\right\rangle U\left(e_{j}\right)$. Therefore for $k \neq i$ 
we have $\left\langle U\left(e_{j}\right), e_{k}\right\rangle=0$, and then $U\left(e_{j}\right)=\nu_{j} e_{i}$, for some modulus 1 complex number $\nu_{j}$. On the other hand, if $T=e_{i} \otimes e_{i}$, we have $T U^{t}\left(e_{k}\right)=\left\langle U^{t}\left(e_{k}\right), e_{i}\right\rangle e_{i}=$ $\pm U T^{t}\left(e_{k}\right)= \pm\left\langle e_{k}, e_{i}\right\rangle U\left(e_{i}\right)$. This implies that $\left\langle U\left(e_{i}\right), e_{k}\right\rangle=0$, for every $k \neq i$. This implies that, for every $i, U\left(e_{i}\right)=\mu_{i} e_{i}$. Then, given $j \neq i$, we have that $U\left(e_{j}\right)=\nu_{j} e_{i}=\mu_{j} e_{j}$, which is impossible. Therefore there exist no $U$ and $V$ (unitary operators) so that $U^{t} V=\alpha I d$, and the equation (2.1) holds for every $T \in \mathcal{I}$.

II. $U^{t} V=\alpha I d+\beta V^{*} U^{* t}(\beta \neq 0)$. We also have that $\alpha U V^{t}=\left(\lambda^{2}+1\right) I d$ and $-\beta U V^{t}=\lambda^{2} V^{* t} U^{*}$. These equations imply that $U^{* t}=-\frac{\beta}{\lambda^{2}} V U^{t} V$, and $\left(1+\frac{\beta^{2}}{\lambda^{2}}\right) U^{t} V=\alpha I d$. We observe that $\alpha=0$; then $\lambda^{2}=-1$ and $\beta= \pm 1$. Therefore $U^{t} V= \pm V^{*} U^{* t},\left(U V^{t}\right)^{2}=I d$, and $\left(U^{t} V\right)^{2}= \pm I d$. As previously considered, we let $T=e_{i} \otimes e_{j}(i \neq j)$; then $(\lambda+1)\left\langle V\left(e_{i}\right), e_{i}\right\rangle U\left(e_{j}\right)=\left\langle U^{t} V\left(e_{i}\right), e_{j}\right\rangle U V^{t}\left(e_{i}\right)$. If there exists $i$ so that $\left\langle V\left(e_{i}\right), e_{i}\right\rangle \neq 0$, then $V^{t}\left(e_{i}\right)=\frac{(\lambda+1)\left\langle V\left(e_{i}\right), e_{i}\right\rangle}{\left\langle U^{t} V\left(e_{i}\right), e_{j}\right\rangle} e_{j}$, for $j \neq i$. Let $\alpha_{j}=\frac{(\lambda+1)\left\langle V\left(e_{i}\right), e_{i}\right\rangle}{\left\langle U^{t} V\left(e_{i}\right), e_{j}\right\rangle}$; then $\left|\alpha_{j}\right| \geq|\lambda+1|\left|\left\langle V\left(e_{i}\right), e_{i}\right\rangle\right| \neq 0$. Then the series $\sum_{k}\left|\left\langle V^{t}\left(e_{i}\right), e_{k}\right\rangle\right|^{2}$ diverges which would contradict Parseval's identity. Therefore, we must have that for every $i,\left\langle V\left(e_{i}\right), e_{i}\right\rangle=0$. This implies that $\left\langle U^{t} V\left(e_{i}\right), e_{j}\right\rangle=0$, for $j \neq i$ and $U^{t} V\left(e_{i}\right)=\alpha_{i} e_{i}$. Since $\left(U^{t} V\right)^{2}= \pm I d$ we have that $\alpha_{i}^{2}= \pm 1$.

We evaluate $\lambda T-(\lambda+1) U T^{t} V+U V^{t} T U^{t} V=O$ with $T=e_{i} \otimes e_{j}$ at $V^{*}\left(e_{i}\right)$, and obtain that

$$
\lambda\left\langle V^{*}\left(e_{i}\right), e_{j}\right\rangle e_{i}-(\lambda+1) U\left(e_{j}\right)+\alpha_{i}\left\langle U\left(e_{j}\right), e_{i}\right\rangle e_{i}=O .
$$

Therefore

$$
\lambda\left\langle V^{*}\left(e_{i}\right), e_{j}\right\rangle\left\langle e_{i}, U\left(e_{j}\right)\right\rangle-(\lambda+1)+\alpha_{i}\left\langle U\left(e_{j}\right), e_{i}\right\rangle\left\langle e_{i}, U\left(e_{j}\right)\right\rangle=0,
$$

which implies that $\left(\lambda \bar{\alpha}_{i}+\alpha_{i}\right)\left|\left\langle e_{i}, U\left(e_{j}\right)\right\rangle\right|^{2}=\lambda+1$, and $\left|\left\langle e_{i}, U\left(e_{j}\right)\right\rangle\right|^{2}=\frac{\lambda+1}{\left.\lambda \bar{\alpha}_{i}+\alpha_{i}\right)}$. Clearly $\lambda \bar{\alpha}_{i}+\alpha_{i} \neq 0$ because $\lambda \neq-1$ and $\alpha_{i}^{2}= \pm 1$. Similarly to the previous case we have $\left|\left\langle U^{*}\left(e_{i}\right), e_{k}\right\rangle\right|$ must converge to zero. Thus, $\alpha=0$ yields no solution and it is left to analyze the case where $\alpha \neq 0$ and $\beta \neq 0$. This will imply that $U V^{t}=\frac{\lambda^{2}+1}{\alpha} I d$ and hence $U^{t} V=\frac{\lambda^{2}+1}{\alpha} I d$. For $T=e_{i} \otimes e_{j}(i \neq j)$, we have that $\lambda T\left(e_{k}\right)-(\lambda+1) U T^{t}\left(V\left(e_{k}\right)\right)+\frac{\lambda^{2}+1}{\alpha} U V^{t} T\left(e_{k}\right)=0$. If $k \neq j$, then $\left\langle V\left(e_{k}\right), e_{i}\right\rangle=0$. This implies that $\left\langle V^{*}\left(e_{i}\right), e_{k}\right\rangle=0$ for all $k$. This contradiction completes the proof.

Proposition 2.3. Let $(\mathcal{I}, \nu)$ be a separable minimal norm ideal different from $\mathcal{C}_{2}$ (the Hilbert-Schmidt class) and let $Q$ be a generalized bi-circular projection on $\mathcal{I}$. If $Q$ is associated with a surjective isometry $\tau$ on $\mathcal{I}$ of the form $\tau(T)=U T V$ ( $U$ and $V$ unitary operators on $\mathcal{H}$ ) and $\lambda \neq-1$, then $Q$ is given as

$$
Q(T)=P_{F} T \text { or } Q(T)=T P_{F},
$$

where $P_{F}$ represents a projection on $\mathcal{H}$ onto a closed subspace $F$.

Proof. Since $Q$ is a generalized bi-circular projection then

$$
Q(T)=\frac{1}{1-\lambda}[-\lambda T+U T V]
$$

and

$$
\lambda T-(\lambda+1) U T V+U^{2} T V^{2}=O
$$

for every $T \in \mathcal{I}$. 
It follows from Fong and Sourour's theorem that $\left\{I d, V, V^{2}\right\}$ must be linearly dependent. Therefore we have two cases to analyze: 1) $V=\alpha I d$ and $V^{2}=\alpha^{2} I d$, and 2) $V^{2}=\alpha I d+\beta V$, for some complex numbers $\alpha$ and $\beta$.

1) If $V=\alpha I d$ and $V^{2}=\alpha^{2} I d$, then $|\alpha|=1$ and $\lambda I d=\alpha(1+\lambda) U-\alpha^{2} U^{2}$. The spectral representation of $U$ is therefore of the form

$$
U=\bar{\alpha} \lambda P_{k e r\{\alpha U-\lambda I d\}}+\bar{\alpha} P_{k e r\{\alpha U-I d\}},
$$

where $P_{k e r}\{\alpha U-\lambda I d\}$ represents the projection onto $\operatorname{ker}\{\alpha U-\lambda I d\}$. Therefore $Q(T)$ $=P_{k e r}\{\alpha U-I d\}$.

2) If $V^{2}=\alpha I d+\beta V$, for some complex numbers $\alpha$ and $\beta$, then $\lambda I d=-\alpha U^{2}$ and $(\lambda+1) U=\beta U^{2}$. This implies that $|\alpha|=1$ and that $\alpha=-\frac{\lambda \beta^{2}}{(\lambda+1)^{2}}$. Therefore the spectral theorem applied to $V$ implies the following representation:

$$
V=\frac{\beta \lambda}{\lambda+1} P_{K \operatorname{er}\left(V-\frac{\beta \lambda}{\lambda+1} I d\right)}+\frac{\beta}{\lambda+1} P_{K e r\left(V-\frac{\beta}{\lambda+1} I d\right)} .
$$

We also notice that $P_{V-\frac{\beta}{\lambda+1} I d}+P_{V-\frac{\beta \lambda}{\lambda+1} I d}=I d$. Therefore, if we denote $\operatorname{Ker}\left(V-\frac{\beta}{\lambda+1} I d\right)$ by $F$, we have that

$$
Q(T)=\frac{1}{1-\lambda}[-\lambda T+U T V]=T P_{F} .
$$

This completes the proof of the proposition.

Corollary 2.4. If $Q(T)=P_{F} T$ or $Q(T)=T P_{F}$, where $P_{F}$ represents a projection on $\mathcal{H}$, then $Q$ is the average of the identity with an isometric reflection.

Proof. We consider $Q(T)=P_{F} T$ and show that $Q$ is the average of the identity with an isometric reflection. If $\lambda$ is a modulus 1 complex number, let $U_{\lambda}$ be defined on $\mathcal{H}$ by $U_{\lambda}(v)=P_{F}(v)+\lambda\left(I d-P_{F}\right)(v)$. It is easy to see that $U_{\lambda}$ is a surjective isometry. In fact, given $v \in \mathcal{H}$ we have that

$$
\left\|U_{\lambda}(v)\right\|^{2}=\left\|P_{F}(v)\right\|^{2}+\left\|\left(I d-P_{F}\right)(v)\right\|^{2}=\|v\|^{2} .
$$

The surjectivity follows, since $U_{\lambda}\left(P_{F}(v)+\lambda^{-1}\left(I d-P_{F}\right)(v)\right)=v$. On the other hand, we have that $\tau(T)=2 P_{F} T-T$ is an isometry of $\mathcal{I}$ since it can be written as $\tau(T)=U_{-1} T I d$. It follows that $Q(T)=\frac{1}{2}(I d+\tau)(T)$. If $Q(T)=T P_{F}$ the proof follows similarly.

Proposition 2.5. Let $(\mathcal{I}, \nu)$ be a separable minimal norm ideal different from $\mathcal{C}_{2}$ (the Hilbert-Schmidt class) and let $Q$ be a generalized bi-circular projection on $\mathcal{I}$. If $Q$ is associated with a surjective isometry $\tau$ on $\mathcal{I}$ of the form $\tau(T)=U T V(U$ and $V$ unitary operators on $\mathcal{H}$ ) and $\lambda=-1$, then $Q$ is the average of the identity with an isometric reflection on $\mathcal{H}$.

Proof. The operator $Q(T)=\frac{1}{2}[T+U T V]$ is a projection if and only if $T=U^{2} T V^{2}$ for every operator $T \in \mathcal{I}$. Therefore we have that $V^{2}=\alpha I d$ and $U^{2}=\bar{\alpha} I d$.

Theorem 2.6. If $(\mathcal{I}, \nu)$ is a separable minimal norm ideal different from $\mathcal{C}_{2}$ (the Hilbert-Schmidt class), then $Q$ is a generalized bi-circular projection on $\mathcal{I}$ if and only if $Q$ is the average of the identity with an isometric reflection.

Proof. If a projection $Q$ is the average of the identity with an isometric reflection, denoted by $R$, then $R=Q-(I d-Q)$ and $Q$ is a generalized bi-circular projection with $\lambda=-1$. Conversely, if $Q$ is a generalized bi-circular projection the statement in this theorem follows from Proposition 2.3, Corollary 2.4, and Proposition 2.5. 
The following corollary is an immediate consequence of the previous results.

Corollary 2.7. Every generalized bi-circular projection on $\mathcal{I}$ is a bi-contractive projection, i.e. $\|P\| \leq 1$ and $\|I d-P\| \leq 1$.

\section{ACKNOWLEDGMENTS}

The authors wish to thank C. K. Li for providing the authors with the manuscript [7] that largely motivated the results in this paper.

\section{REFERENCES}

[1] Behrends, E., M-Structure and the Banach-Stone Theorem, Lecture Notes in Mathematics 736 (1979), Springer-Verlag. MR547509 (81b:46002)

[2] Berkson, E., Hermitian projections and orthogonality in Banach spaces, Proc. London Math. Soc. 24:3 (1972), 101-118. MR0295123 (45:4191)

[3] Bernau, S. J. and Lacey, H. E., Bicontractive projections and reordering of $L_{p}-$ spaces, Pac. Journal of Math. 69:2 (1977), 291-302. MR0487416 (58:7054)

[4] Botelho, F. and Jamison, J. E., Generalized bi-circular projections on $\mathcal{C}(\Omega, X)$, Rocky Mountain Journal (to appear).

[5] Friedman, Y. and Russo, B., Contractive projections on $C_{0}(K)$, Transactions of the AMS 273:1 (1982), 57-73. MR664029 (83i:46062)

[6] Fleming, R. and Jamison, J., Isometries on Banach Spaces (2003), Chapman \& Hall.

[7] Fosner, M.,Ilisevic, D. and Li, C., G-invariant norms and bicircular projections, Linear Algebra and Applications 420 (2007), 596-608. MR2278235

[8] Groot, J. and Wille, R., Rigid continua and topological group-pictures, Arch. Math. IX (1958), 441-446. MR0101514 (21:324)

[9] Jamison, J. E., Bicircular projections on some Banach spaces, Linear Algebra and Applications 420 (2007), 29-33. MR2277626

[10] Lindenstrauss, J. and Tzafriri, L., Classical Banach Spaces I (1977), Springer Verlag. MR0500056 (58:17766)

[11] Shatten, R., Norm Ideals of Completely Continuous Operators (1970), Springer-Verlag, Berlin. MR0257800 (41:2449)

[12] Sourour, A. R., Isometries of norm ideals of compact operators, J. Funct. Anal. 43 (1981), 69-77. MR639798 (84e:47061)

[13] Spain, P. G., Ultrahermitian projections on Banach spaces, preprint (2006).

[14] Taylor, A. E., Introduction to Functional Analysis (1957), John Wiley \& Sons Inc. MR0098966 (20:5411)

[15] Fong, C. K. and Sourour, A. R., On the operator identity $\sum A_{k} X B_{k}=0$, Can. J. Math., XXXI (1979), 845-857. MR540912 (80h:47020)

[16] Randrianantoanina, B., Norm-One projections in Banach spaces, Taiwanese Journal of Mathematics. 5:1 (2001), 35-95. MR1816130 (2003d:46001)

[17] Stachó, L. L. and Zalar, B., Bicircular projections on some matrix and operator spaces, Linear Algebra and Applications 384 (2004), 9-20. MR2055340 (2005a:46146)

[18] Stachó, L. L. and Zalar, B., Bicircular projections and characterization of Hilbert spaces, Proc. Amer. Math. Soc. 132 (2004), 3019-3025. MR2063123 (2005b:46152)

Department of Mathematical Sciences, The University of Memphis, Memphis, TenNESSEE 38152

E-mail address: mbotelho@memphis.edu

Department of Mathematical Sciences, The University of Memphis, Memphis, TenNESSEE 38152

E-mail address: jjamison@memphis.edu 